\title{
Nutzung von Lagerbeständen für die Überbrückung der Reaktionszeit zur Nutzung von Kapazitätsflexibilität
}

\author{
DiPL.-WIRTSCH.-ING. STEPHAN MEERS \\ DiPL.-WIRTSCH.-ING. PATRICK PRÜSSING \\ DR.-ING. DIPL.-OEC. ROUVEN NICKEL \\ InSTITUt FÜR INTEGRIERTE PRODUKTION HANNOVER GGMBH, HOLLERITHALLEE 6, 30419 HANNOVER
}

\begin{abstract}
Zusammenfassung
Kurzfristige Kundenbedarfserhöhungen können in einer ausgelasteten Fertigung zu Kapazitätsengpässen führen. In vielen Unternehmen besteht die Möglichkeit, entstehende Engpässe durch Kapazitätsflexibilität auszugleichen. Entsprechende Maßnahmen sind jedoch mit einer Reaktionszeit verbunden. Im Rahmen des von der Deutschen Forschungsgemeinschaft (DFG) geförderten Projektes „Dynamische Losgrößen als Basis einer Methode zur Fertigungssteuerung“ (WI 377/63-1) ist eine Methodik entwickelt worden, die Reaktionszeit für die Nutzung flexibler Kapazitäten durch einen kontrollierten, artikelübergreifenden Abbau von Lagerbeständen zu überbrücken. Die im Artikel beschriebenen Verfahrensschritte stellen die Grundlage zur Implementierung der Methodik in ERP-Systeme dar.
\end{abstract}

\begin{abstract}
Short-term customer demand rises can lead to capacity bottlenecks in a fully occupied production. In many enterprises the possibility exists to compensate arising bottlenecks by employing capacity flexibility. Corresponding measures are, however, connected to a reaction time. In the context of a project sponsored by the German Research Foundation a method has been developed to bridge the reaction time by a controlled reduction of stock levels. The steps described in the article are the basis for an implementation of the method in ERP systems.
\end{abstract}

\section{Ausgangssituation}

In einer Fertigung, in der nicht alle Planungsgrößen (z. B. Kundennachfrage über der Zeit, Durchlaufzeit eines Produktionsauftrages) sicher prognostiziert werden können, dient in der Regel ein Fertigwarenlager zur zeit- und mengenmäßigen Entkopplung von Fertigung und Kunden (Make-to-stock-Fertigung). Auf diese Weise kann schlecht prognostizierbaren Planungsgrößen in der Produktionsplanung begegnet werden [Temp-04]. Wie stark die Schwankungen von Planungsgrößen sein können, die durch das Lager abgefangen werden können, hängt von der Höhe des im Fertigwarenlager vorgehaltenen Sicherheitsbestands ab. Zu starke Planabweichungen in der Fertigung oder kurzfristige Erhöhungen der Kundennachfrage können zu einer „Out-of-stock-Situation“ durch Aufbrauchen des Sicherheitsbestandes führen. Um z. B. im Fall kurzfristig gestiegener Kundennachfragen eine derartige Situation zu vermeiden, bestehen die folgenden Möglichkeiten:

1) Fertigung der Zusatzmengen in einem oder mehreren zusätzlichen Fertigungsaufträgen (Erhöhung der Anzahl von Fertigungsaufträgen),

2) mengenmäßige Anpassung von Fertigungsaufträgen (Erhöhung der Fertigungslosgröße),

3) zeitmäßige Anpassung von Fertigungsaufträgen (Verringerung der Losfrequenz).

Die drei genannten Möglichkeiten werden in gängigen EDV-Systemen zur Produktionsplanung und -steuerung nicht als integrierte Funktionalität abgebildet. Dieser Mangel begründet sich durch das Fehlen eines entsprechenden, softwaretechnisch umsetzbaren Algorithmus zur kurzfristigen Veränderung von Losgrößen und -abständen im Rahmen der Auftragserzeugung und -freigabe. Die Folge ist ein hoher manueller Steuerungsaufwand in der Fertigung. Gängige Produktionsplanungs- und steuerungssysteme (PPS-Systeme) verwenden statische und dynamische Losgrößenverfahren, jedoch können einmal berechnete Losgrößen und daraus abgeleitete Losabstände nicht in einem automatisierten Prozess durch das PPS-System angepasst werden. Für die Losgrößenberechung von Zusatzaufträgen eignen sich tendenziell statische (z. B. Andler-Methode [Andler-29]) und für die periodenweise Anpassung von Losgrößen dynamische Losgrößenbestimmungsverfahren (z. B. Wagner-Within-Alghorithmus [WagWith-58]). Während statische Verfahren von einer konstanten Nachfrage über alle Perioden innerhalb des Planungszeitraums ausgehen, 
werden bei dynamischen Verfahren die Nachfragemengen als schwankend angenommen und mit spezifischen Werten für jede Periode innerhalb des Planungshorizonts beschrieben [Temp-95; Der-95].

Das Erzeugen eines Zusatzauftrages, der die erhöhte Nachfrage abdeckt, bedeutet einen geringeren dispositiven Aufwand, als die mengen- oder zeitmäßige Anpassung mehrerer Aufträge. Jedoch erhöhen sich über der Zeit die Auftragswechselkosten aufgrund der insgesamt erhöhten Anzahl an Fertigungsaufträgen.

Abhängig vom Ausmaß der Nachfrageerhöhung kann der Fall eintreten, dass für die erhöhten Fertigungsmengen nicht genügend Kapazität zur Verfügung steht. Zu geringe Kapazitäten führen in Kombination mit aufgebrauchten Sicherheitsbeständen zu einer Verschlechterung der Liefertreue gegenüber Kunden. Um derartige Engpässe zu vermeiden, können viele Unternehmen ihre Produktionskapazitäten für bestimmte Zeiträume dynamisch anpassen (Kapazitätsflexibilität). Die Maßnahmen der Kapazitätsflexibilität sind vielfältig und reichen von der Einführung zusätzlicher Schichten bis hin zur Einstellung von Zeitarbeitern. In der Regel sind derartige Maßnahmen jedoch mit einem definierten zeitlichen Vorlauf, der so genannten Reaktionszeit verbunden [Breit-00]. In vielen Fällen verschlechtert sich die logistische Leistung der Fertigung bereits während der Reaktionszeit.

\section{Methodik zur artikelübergreifenden Verschiebung von Produktionsmengen}

\subsection{Grundidee}

Am IPH - Institut für Integrierte Produktion Hannover gemeinnützige GmbH wurde im Rahmen des DFGForschungsprojektes „Dynamische Losgrößen als Basis einer Methode zur Fertigungssteuerung“ (WI 377/63-1) eine Methodik entwickelt, mit dem die Reaktionszeit durch eine kurzfristige, artikelübergreifende Verringerung von Produktionsmengen überbrückt und die in PPS-Systemen implementiert werden kann. In den meisten Fällen entstehen bei Kapazitätsengpässen nicht für alle Artikel terminkritische Situationen. So existieren Artikel mit niedrigen, bei steigender Nachfrage bis zur Wiederauffüllung des Lagers zu kurzen Lagerreichweiten (im Folgenden als terminkritische Artikel bezeichnet) und Artikel mit bis zum Wiederauffüllungszeitpunkt ausreichender Lagerreichweite (im Folgenden als Artikel mit Bestandsdeckung bezeichnet). Die hier vorgestellte Methodik nutzt vorhandene Lagerbestände (inkl. Sicherheitsbestände), um kurzfristig durch reduzierte Fertigungsmengen von Aufträgen der Artikel mit Bestandsdeckung freie Kapazitäten für terminkritische Fertigungsaufträge zu generieren. Die Basis dafür liegt auf dem Grundgedanken, dass die Inanspruchnahme von Produktionskapazitäten innerhalb des Artikelspektrums umverteilt werden kann. Artikel mit Bestandsdeckung werden in geringerer Stückzahl gefertigt, um Kapazitäten zur Fertigung der terminkritischen Artikel ohne Bestandsdeckung zu gewinnen.

\subsection{Verfahrensschritte}

Abbildung 1 zeigt die Verfahrensschritte der Methodik. Betrachtungsgegenstand ist die Kapazität desjenigen Arbeitssystems, welches für die betrachtete Planungsperiode das Engpasssystem darstellt. Die Methodik muss ggf. mehrfach durchlaufen werden, da je Durchgang sukzessiv weitere Kapazität für ein Engpassarbeitssystem gewonnen wird. Ein mehrfacher Durchlauf durch ein PPS-System stellt eine artikelübergreifende Liefermengenund termintreue sicher. 


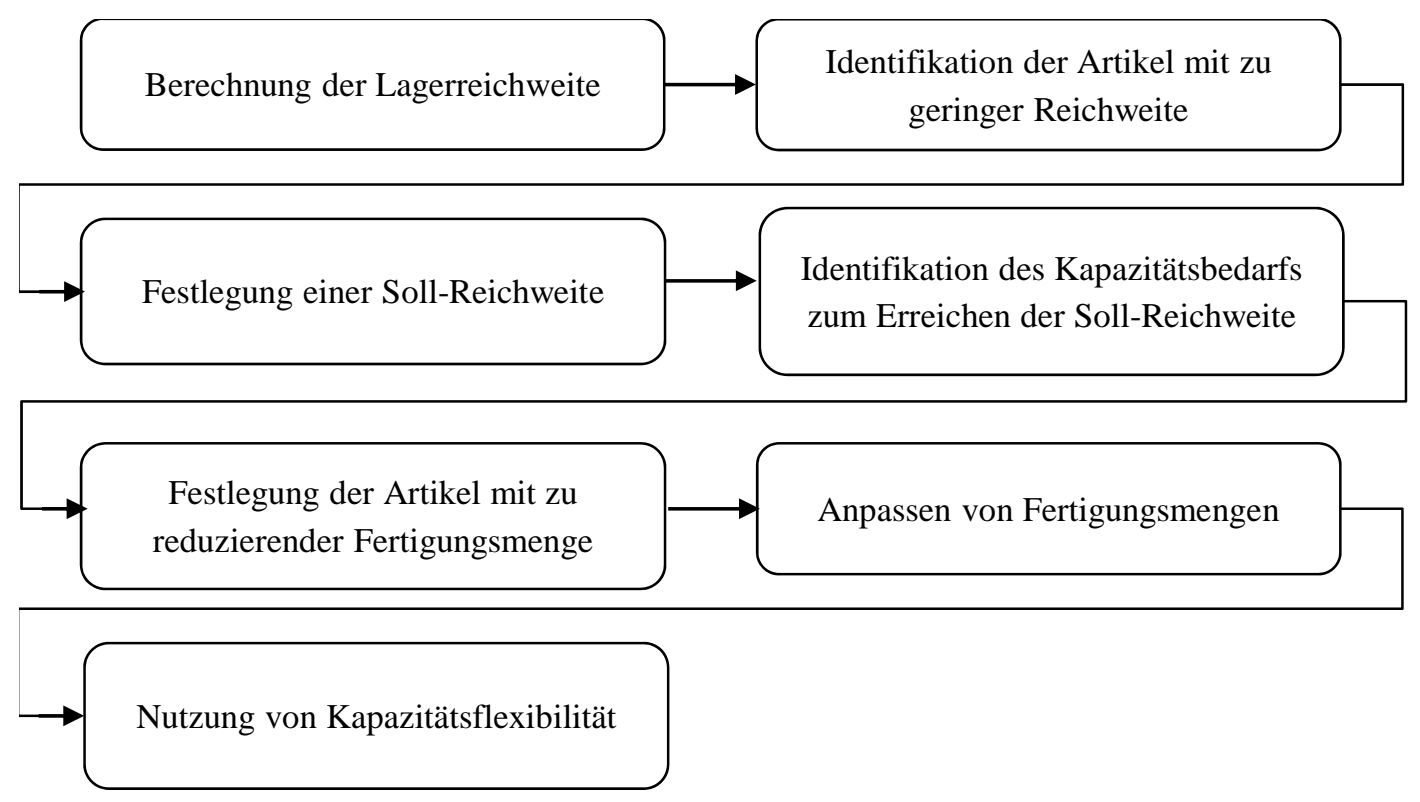

Abbildung 1: Verfahrensschritte

Die Methodik führt insgesamt zu einem Abbau der Bestände im Fertigwarenlager. Daher muss die Anwendung der Prozessschritte mit der Nutzung von Kapazitätsflexibilität (z. B. durch Inanspruchnahme von zusätzlichen Schichten) abschließen, um Sicherheitsbestände artikelübergreifend wiederherzustellen. Im Folgenden werden die Verfahrensschritte der Methodik detailliert beschrieben, wobei beispielhaft von einer artikelübergreifenden Losgrößenanpassung ausgegangen wird.

\section{Verfahrensschritt 1: Berechnung der Lagerreichweite}

Für alle Artikel aus dem Produktionsprogramm wird die Reichweite des Fertigwarenlagerbestandes jeweils gemäß Formel (1) berechnet:

$\mathrm{R}_{\text {Artikel }}=\frac{\text { durchschnittlicher Lagerbestand pro Periode[Stück] }}{\text { durchschnittlicher Bedarf pro Periode[Stück/BKT] }}$

mit

$\mathrm{R}_{\text {Artikel }} \quad$ Lagerreichweite des Artikels [BKT]

Abbildung 2 zeigt ein Lagerdiagramm, in dem eine Situation dargestellt ist, in der die Reaktionszeit zur Installation zusätzlicher Kapazitäten zur Produktion für eine vollumfängliche Bedarfsdeckung nicht ausreicht. Artikelzu- und -abgang sind kumuliert und idealisiert dargestellt. Der rote Graph zeigt die im zeitlichen Verlauf abnehmende Lagerbestandshöhe und schneidet an der Stelle die Abszisse, an der die Zu- und Abgangsgeraden ihren Schnittpunkt besitzen. Zu diesem Zeitpunkt ist der Lagerbestand inklusive des Sicherheitsbestands erschöpft, so dass im Rahmen der Reaktionszeit zur Installation zusätzlicher Kapazitäten eine Antizipation von Nachfrageengpässen nicht möglich ist. Zur Vereinfachung der Darstellung wird in dieser Ausführung von einer arbeitssystem- und artikelunspezifischen Reaktionszeit für die Installation von Zusatzkapazitäten ausgegangen. Für das Verfahren können jedoch auch artikel- und arbeitssystemspezifische Reaktionszeiten als Eingangsgrößen verwendet werden. 


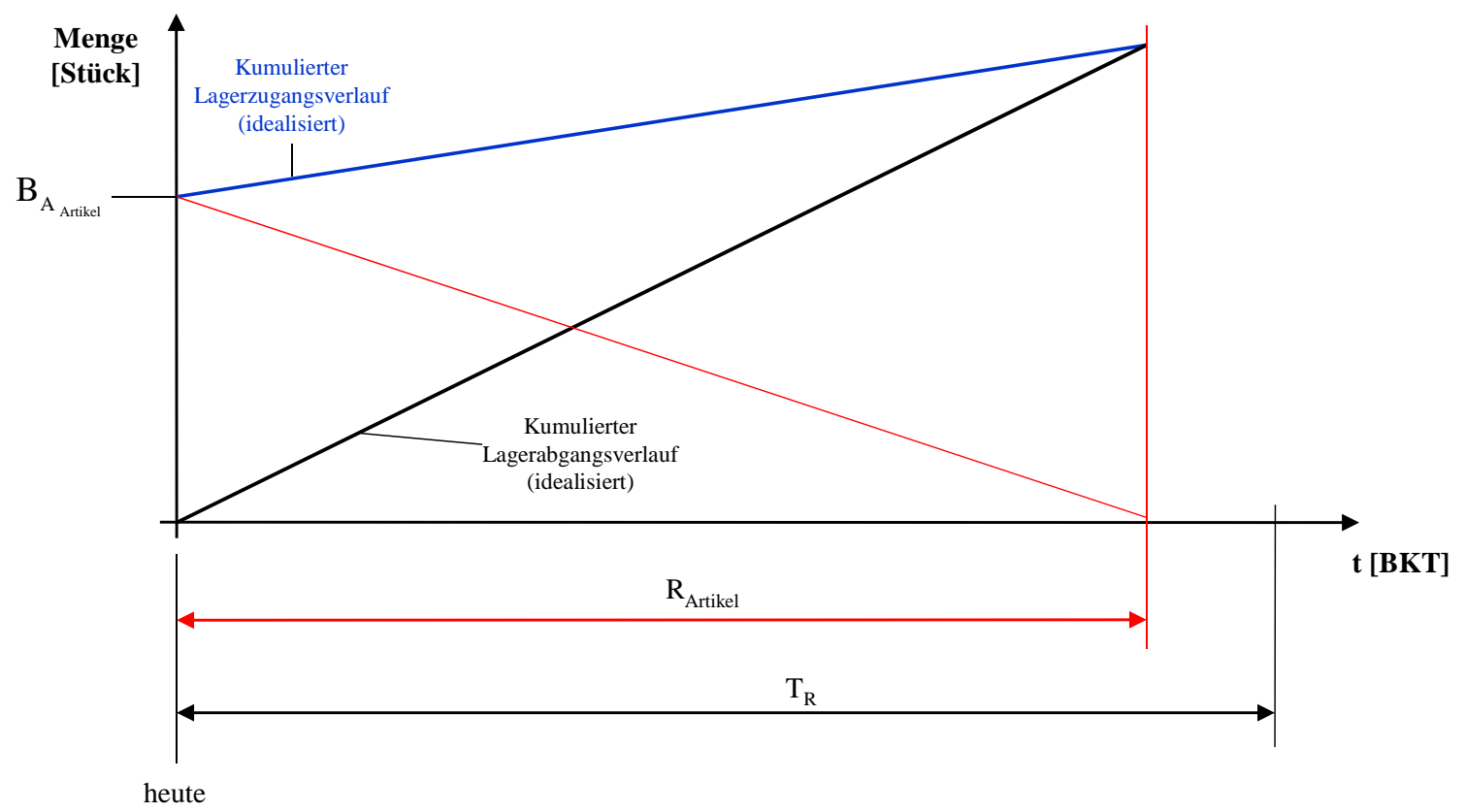

Legende:

$\mathrm{B}_{\mathrm{A}_{\text {Artikel }}}$ - Anfangsbestand des Artikels

$\mathrm{R}_{\text {Artikel }}$ - Reichweite des Artikels

$\mathrm{T}_{\mathrm{R}}$ - Reaktionszeit

Abbildung 2: Lagerdiagramm mit Artikelreichweite

\section{Verfahrensschritt 2: Identifikation von Artikeln mit zu geringer Reichweite}

Aus den in Verfahrensschritt 1 berechneten Lagerreichweiten werden diejenigen als kritische Artikel identifiziert, bei denen die Lagerreichweite für eine pünktliche und vollständige Bedarfsdeckung kürzer als die Reaktionszeit zur Installation von Zusatzkapazitäten am Engpasssystem ist. Als kritisch gilt ein Artikel dann, wenn sein Lagerbestand vor dem Ablauf der Reaktionszeit erschöpft sein wird. Die identifizierten Artikel werden nach absteigender Differenz zwischen Reaktionszeit und Lagerreichweite sortiert, um eine Rangfolge kritischer Artikel zu erheben und somit eine Priorisierung für die Reichweitenanpassung zu erhalten.

\section{Verfahrensschritt 3: Festlegung einer Soll-Reichweite}

Für die in Verfahrensschritt 2 identifizierten Artikel mit kritischer Reichweite wird in diesem Verfahrensschritt eine Soll-Reichweite festgelegt. Diese ist so festzulegen, dass der Lagerbestand frühestens zum Ende der Reaktionszeit erschöpft sein wird.

Abbildung 3 zeigt beispielhaft die idealisierten kumulierten Lagerzu- und -abgangsverläufe für einen Artikel im Kontext von Soll-Reichweite und Reaktionszeit. 


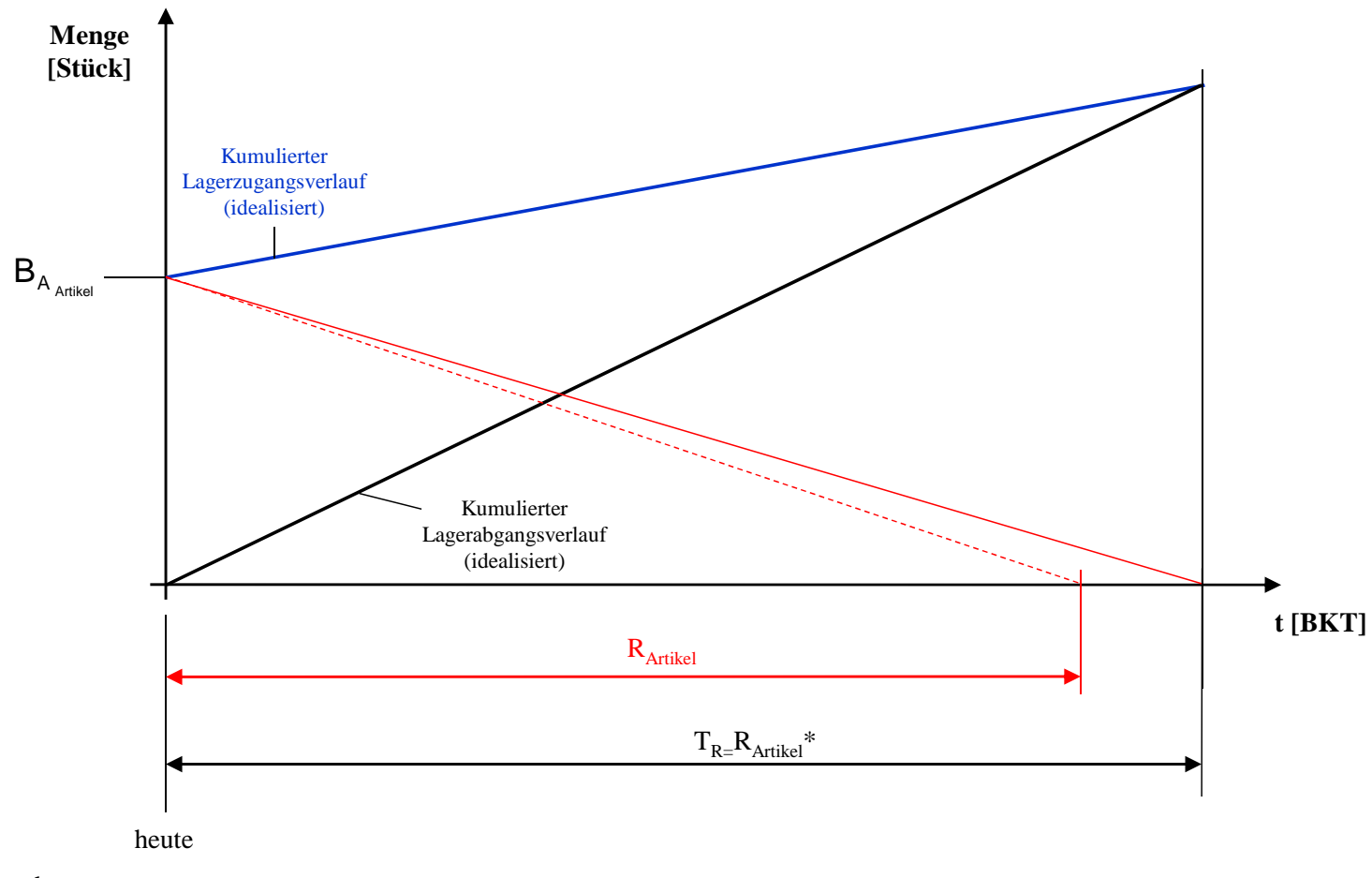

Legende:

$\mathrm{B}_{\mathrm{A}_{\text {Artikel }}}$ - Anfangsbestand des Artikels

$\mathrm{R}_{\text {Artikel }}$ - Reichweite des Artikels

$\mathrm{R}_{\text {Artikel }} *$-Sollreichweite des Artikels

$\mathrm{T}_{\mathrm{R}}$ - Reaktionszeit

Abbildung 3: Lagerdiagramm mit definierter Soll-Reichweite

\section{Verfahrensschritt 4: Identifikation des Kapazitätsbedarfs zum Erreichen der Soll-Reichweite}

Im vierten Verfahrensschritt wird für die terminkritischen Artikel der Kapazitätsbedarf ermittelt, der für die Herstellung der Fertigungsmenge zum Erreichen der Soll-Reichweite erforderlich ist. Der Kapazitätsbedarf ergibt sich aus der Fertigungsmenge multipliziert mit der Stückzeit plus einer losgrößenabhängigen Rüsthäufigkeit multipliziert mit der Rüstdauer. Abbildung 4 zeigt im Lagerdiagramm die zusätzlich zu fertigende Menge zur Sicherstellung der Lieferfähigkeit. Die gestrichelte blaue Linie zeigt den Lagerzugangsverlauf in der Ausgangssituation. Die gestrichelte rote Linie den ursprünglichen Lagerabgangsverlauf, dessen Schnittpunkt mit der Abszisse die Lagerreichweite der Ausgangssituation markiert. 


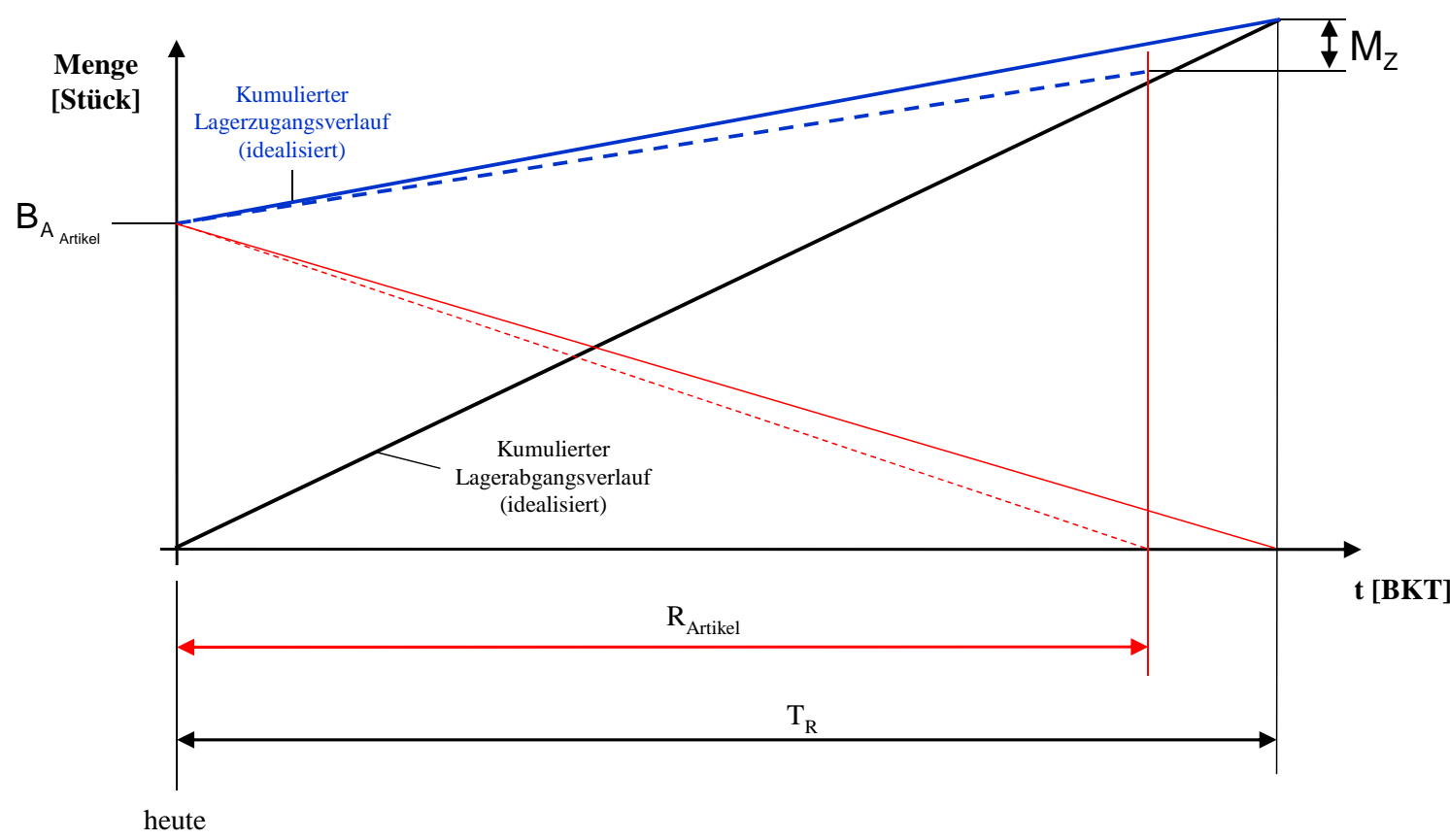

Legende:

$$
\begin{aligned}
& \mathrm{B}_{\mathrm{A}_{\text {Artikel }}} \text { - Anfangsbestand des Artikels } \\
& \mathrm{R}_{\text {Artikel }} \text { - Reichweite des Artikels } \\
& \mathrm{M}_{\mathrm{Z}} \text { - zusätzlich zu fertigende Menge } \\
& \mathrm{T}_{\mathrm{R}} \text { - Reaktionszeit }
\end{aligned}
$$

Abbildung 4: Zu fertigende Zusatzmenge

Die Berechnung des zusätzlichen Kapazitätsbedarfs an einem Arbeitssystem für den terminkritischen Artikel erfolgt gemäß folgender Formel:

$\mathrm{KAP}_{\text {zus B }}=\mathrm{M}_{\mathrm{z}} \cdot \mathrm{t}_{\mathrm{e}}+\mathrm{t}_{\mathrm{r}}$

mit

$\mathrm{KAP}_{\text {ZUSB }}$

$\mathrm{M}_{\mathrm{Z}}$

$t_{e}$

$t_{r}$
Zusätzlicher Kapazitätsbedarf [ZE]

Zusätzlich zu fertigende Menge [ME]

Einzelstückzeit [ZE/ME]

Rüstzeit je Arbeitsvorgang [ZE]

Ergebnis der Rechnung ist der zusätzliche Kapazitätsbedarf bis zum Ende der Reaktionszeit. Dieser Kapazitätsbedarf muss durch eine Freisetzung von Fertigungskapazität bei nicht kritischen Artikeln generiert werden, um die artikelübergreifende Kundentermineinhaltung sicherstellen zu können.

Bei welchen Artikeln die erforderliche Kapazität freigesetzt werden kann, wird im nächsten Verfahrensschritt bestimmt.

\section{Verfahrensschritt 5: Festlegung der Artikel mit zu reduzierender Fertigungsmenge}

Nachdem die freizusetzende Kapazität zur ausreichenden und pünktlichen Bedarfsdeckung terminkritischer Artikel bekannt ist, müssen im nächsten Schritt der bzw. die Artikel festgelegt werden, deren Fertigungsmenge zur Freisetzung von Kapazität reduziert werden kann. Dazu wird für jeden nicht kritischen Artikel berechnet, wie viel Kapazität (gemessen in Vorgabestunden) freigesetzt werden kann, wenn die Lagerreichweite auf das erforderliche Mindestniveau zum Erreichen des Endes der Reaktionszeit reduziert wird. Abbildung 5 verdeutlicht das dem Verfahren zu Grunde liegende Prinzip verringerter Produktionsmengen. Die idealisierte Gerade des Lagerzugangs verläuft flacher. In der Folge treffen Lagerzu- und abgangsgeraden aufeinander, der artikelspezifische Lagerbestand ist erschöpft. Dies geschieht zu dem Zeitpunkt, an dem die Reaktionszeit beendet ist. 


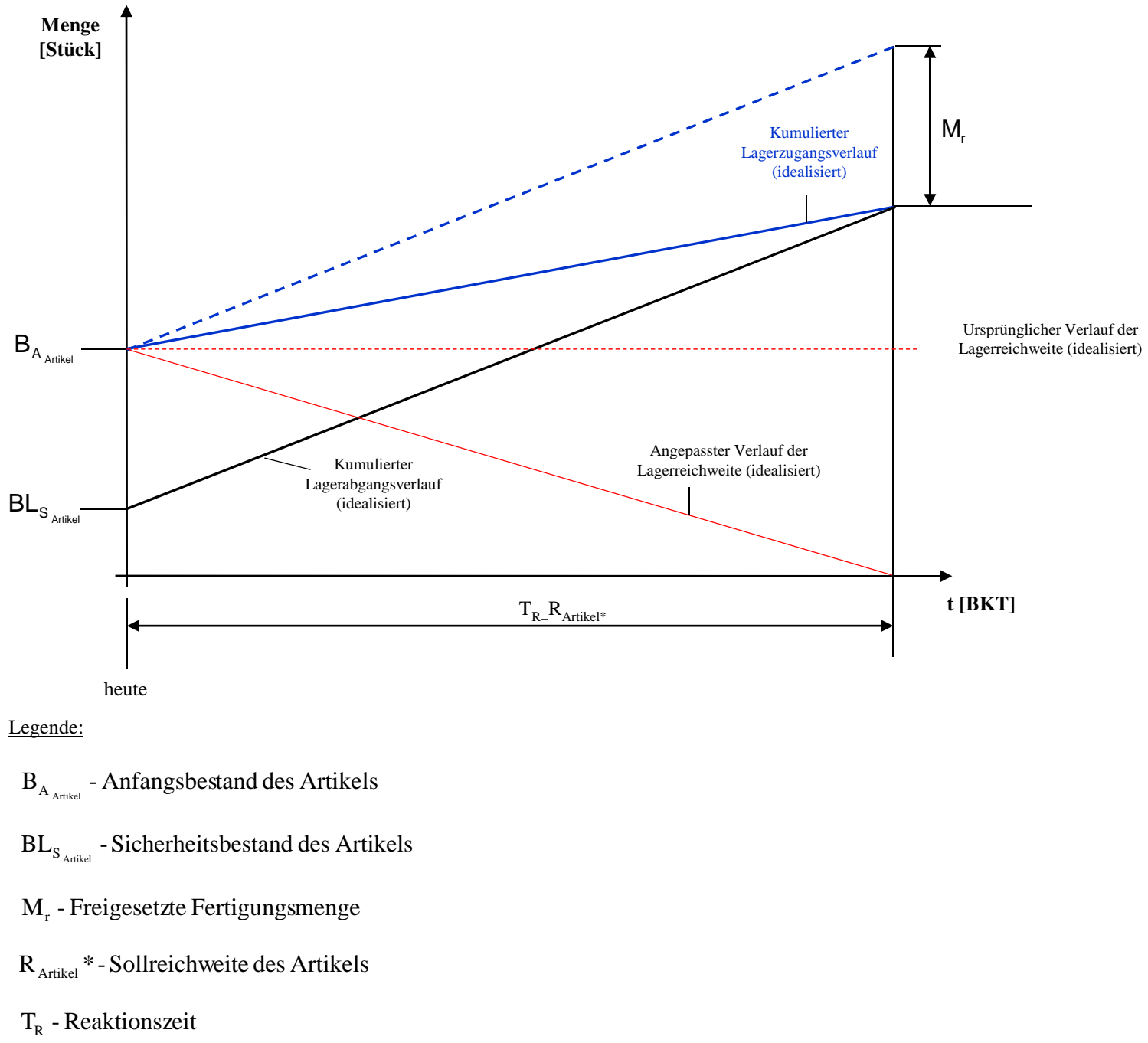

Abbildung 5: Reduzierte Fertigungsmenge

Die Berechnung der freisetzbaren Kapazität pro Artikel erfolgt anhand folgender Formel:

$\mathrm{KAP}_{\text {umver }}=\mathrm{M}_{\mathrm{R}} * \mathrm{t}_{\mathrm{e}}+\mathrm{t}_{\mathrm{r}}$

mit

$\mathrm{KAP}_{\text {umver }} \quad$ Durch Umverteilung temporär gewonnene Kapazität [ZE]

$\mathrm{M}_{\mathrm{R}} \quad$ Subtrahend der Fertigungsmenge [ME]

$\mathrm{t}_{\mathrm{e}} \quad$ Stückzeit [ZE/ME]

$\mathrm{t}_{\mathrm{r}} \quad$ Rüstzeit je Arbeitsvorgang [ZE]

Ergebnis dieses Verfahrensschrittes ist eine Sammlung derjenigen Artikel, über deren Reduzierung der Fertigungsmenge und damit Verkürzung der Lagerreichweite Kapazität für die Fertigung der terminkritischen Artikel freigesetzt werden kann.

\section{Verfahrensschritt 6: Anpassen der Losgröße}

In diesem Beispiel werden die Losgrößen der in Verfahrensschritt 5 identifizierten Artikel gegenüber der ursprünglich berechneten, wirtschaftlichen Fertigungslosgröße verringert. Für eine derartige Losgrößenreduzierung muss je Artikel das Arbeitssystem identifiziert werden, an dem am wenigsten Kapazität freigesetzt werden kann. Für dieses Arbeitssystem wird die freisetzbare Kapazität in Stück umgerechnet. Um diese Größe kann das entsprechende Fertigungslos maximal reduziert werden. 


\section{Verfahrensschritt 7: Nutzung von Kapazitätsflexibilität}

Durch die im Rahmen der Methodik zu durchlaufenden Verfahrensschritte wird der Bestand der Artikel mit Bedarfsdeckung

- abhängig vom Ausmaß der Losgrößen- bzw. Losabstandsanpassung - ggf. erheblich reduziert. Daher muss möglichst zeitnah flexible Zusatzkapazität installiert werden, um neben der Herstellung zukünftig benötigter Bedarfsmengen die erforderlichen Bestände wiederherzustellen. Nach Ablauf der Reaktionszeit müssen demnach unmittelbar Maßnahmen der Kapazitätsflexibilität eingeleitet werden und so lange aufrecht erhalten bleiben, bis die erforderlichen Bestände in allen Lägern wieder aufgebaut sind.

\section{Fazit}

Abhängig davon, wie die erhöhte Fertigungsmenge realisiert werden soll, setzt die Methodik in der Auftragserzeugung oder Auftragsfreigabe an und kann somit direkt in entsprechende Module von PPS-Software integriert werden (Verfahrensschritte 1 bis 6). Während die Auftragserzeugung die Losgrößen festlegt, bestimmt die Auftragsfreigabe über den Startzeitpunkt der Fertigungsaufträge. [Lödding-08]. Das Verfahren kann in gleicher Weise bei Störungen angewendet werden, die zu temporären Kapazitätsreduzierungen und damit zu Engpässen führen können. Durch die in diesem Artikel beschriebene Methodik wird das Problem temporär zu geringer Kapazitäten nicht an der Wurzel gelöst, sondern die Symptome behandelt. Jedoch kann bis zum Aufbau zusätzlicher Kapazität in vielen Fällen Zeit für die Aufrechterhaltung der logistischen Leistung der Fertigung gewonnen werden. Eine Implementierung von Verfahrensschritt 7 in PPS-Systemen im Kontext der Kapazitätssteuerung scheint darüber hinaus denkbar.

Die Autoren danken der Deutschen Forschungsgemeinschaft (DFG) für die finanzielle Unterstützung zur Durchführung des Vorhabens WI 377/63-1.

\section{Literatur}

\begin{tabular}{|l|l|}
\hline [Temp-04] & $\begin{array}{l}\text { Günther, H.-O.; Tempelmeier, H.: Produktion und Logistik. 6. Auflage, Springer-Verlag } \\
\text { Berlin u. a. 2004. }\end{array}$ \\
\hline [Andler-29] & $\begin{array}{l}\text { Andler, K.: Rationalisierung der Fabrikation und optimale Losgröße. R. Oldenbourg } \\
\text { Verlag, München, 1929. }\end{array}$ \\
\hline [WagWith-58] & $\begin{array}{l}\text { Wagner, H.; Within, T.: Dynamic version of the economic lot size model. In: Management } \\
\text { Science, vol. 5 (1958), no. 5, pp. 89-96. }\end{array}$ \\
\hline [Temp-95] & $\begin{array}{l}\text { Tempelmeier, H.: Material-Logistik - Modelle und Algorithmen für die } \\
\text { Produktionsplanung und -steuerung in Advanced Planning-Systemen. 7. Auflage, } \\
\text { Springer-Verlag, Heidelberg, 1995. }\end{array}$ \\
\hline [Der-95] & $\begin{array}{l}\text { Derstroff, M.: Mehrstufige Losgrößenplanung mit Kapazitätsbeschränkungen. Physica- } \\
\text { Verlag, Heidelberg, 1995. }\end{array}$ \\
\hline [Breit-00] & $\begin{array}{l}\text { Breithaupt, J.-W.: Rückstandorientierte Produktionsregelung von Fertigungsbereichen, } \\
\text { Dissertation, Universität Hannover. Fortschritt-Berichte VDI, Reihe 2, Nr. 571, VDI- } \\
\text { Verlag, Düsseldorf, 2000. }\end{array}$ \\
\hline [Lödding-08] & $\begin{array}{l}\text { Lödding, H.: Verfahren der Fertigungssteuerung. 2. Auflage, Springer-Verlag, Berlin u. a. } \\
\text { 2008. }\end{array}$ \\
\hline
\end{tabular}

